\title{
Calidad de control metabólico en pacientes ambulatorios con diabetes tipo 2 atendidos en una clínica privada
}

\section{Quality of metabolic control in outpatients with type 2 diabetes seen in a private clinic}

Correspondencia María Lazo-Porras maria.lazo@upch.pe

Recibido: 27/03/2017

Arbitrado por pares

Aprobado: 07/06/2017

Citar como: Azañedo D, BendezúQuispe G, Lazo-Porras $M$, Cárdenas-Montero D, Beltrán-Ale $G$, Thomas NJ, et al. Calidad de control metabólico en pacientes ambulatorios con diabetes tipo 2 atendidos en una clínica privada. Acta Med Peru. 2017;34(2):106-13
Diego Azañedo 1,a, Guido Bendezú-Quispe ${ }^{2, b}$, María Lazo-Porras ${ }^{3,4, b}$, Deborah Cárdenas-Montero ${ }^{3, b}$, Guillermo Beltrán-Ale ${ }^{3,5, b}$, Nimmy J. Thomas ${ }^{6, b}$, Ruben M. Ceballos ${ }^{3, c}$, Germán Málaga $3,4,7, b$

1 Centro de Estudios de Población, Universidad Católica los Ángeles de Chimbote. Chimbote, Perú.

2 Facultad de Salud Pública y Administración, Universidad Peruana Cayetano Heredia. Lima, Perú.

3 CONEVID Unidad de Conocimiento y Evidencia, Universidad Peruana Cayetano Heredia. Lima, Perú.

4 CRONICAS Centre of Excellence in Chronic Diseases, Universidad Peruana Cayetano Heredia. Lima, Perú.

5 Department of pediatrics, Cincinnati Children's Hospital Medical Center. Cincinnati, Ohio, United States.

6 Rollins School of Public Health, Emory University. Atlanta, GA, United States.

7 Facultad de Medicina, Universidad Peruana Cayetano Heredia. Lima, Perú.

a Cirujano dentista, b Médico cirujano, c Estudiante de Medicina

\section{RESUMEN}

Objetivo: Evaluar la calidad de control metabólico en pacientes ambulatorios con diabetes mellitus tipo 2 (DM2) de una clínica privada en Lima, Perú. Materiales y métodos: Estudio de corte transversal en la consulta externa del Servicio de endocrinología de una clínica privada de Lima, Perú. Se recolectó información socio demográfica, historia de enfermedad, autorreporte de complicaciones y comorbilidades, medidas antropométricas y presión arterial. Además, se tomó muestras de sangre para análisis de prueba rápida de glucosa, hemoglobina glicosilada (HbA1c) y perfil lipídico. Se consideró pobre control metabólico si el participante tenía HbA1c $>7 \%$, colesterol LDL (LDL-c) $\geq 100 \mathrm{mg} / \mathrm{dl}$, y presión arterial $\geq 130 / 80 \mathrm{mmHg}$. Se aplicaron cuestionarios adicionales, para medir la adherencia al tratamiento, actividad física, calidad de vida, autoeficacia y depresión. Resultados: Se incluyó a 60 participantes, $53,3 \%(32 / 60)$ fueron de sexo femenino y la media de edad fue $63,1 \pm 13,2$ años. Se encontró pobre control metabólico en $85 \%$ (51/60) de los participantes; presión arterial controlada en $71,2 \%$ (42/60), LDL-c controlado en $10 \%$ (6/60) y HbA1c controlado en $48,3 \%$ (29/60) de los participantes respectivamente. Complicaciones crónicas como retinopatía se autorreportó en el 3,3\% (2/60) participantes, neuropatía en $10 \%(6 / 60)$, nefropatía en 1,7\% (1/60), presión arterial alta en $30 \%(18 / 60)$ y enfermedad cerebro vascular en $5 \%(3 / 60)$ de los participantes. Conclusión: La prevalencia de control metabólico no controlado es elevada, a pesar del contexto de atención en una clínica privada. Regulación y medidas para mejorar el control en centros privados es necesario.

Palabras clave: Diabetes mellitus tipo 2; Instituciones privadas de salud; Metabolismo (fuente: DeCS BIREME). 


\begin{abstract}
Objective: To assess the quality of metabolic control among outpatients with type 2 diabetes mellitus (DM2) in a private clinic in Lima, Peru. Materials and methods: This is a cross-sectional study in the outpatient endocrinology service of a private clinic in Lima, Peru. Socio-demographic information, history of diabetes, self-report of complications and comorbidities, anthropometric measures and blood pressure data were collected. Blood samples were taken for assessing fasting blood glucose, glycated hemoglobin ( $\mathrm{HbA1c}$ ) and lipid profile. A poor metabolic control was considered if the participant had $\mathrm{HbA1c}$ $>7 \%$, LDL cholesterol (LDL-c) $\geq 100 \mathrm{mg} / \mathrm{dl}$, and blood pressure $\geq 130 / 80 \mathrm{mmHg}$. Additional questionnaires were administered for measuring adherence to therapy, physical activity, quality of life, self-efficacy and depression. Results: Sixty participants were included; $53.3 \%$ (32/60) were female and their mean age was $63.1 \pm 13.2$ years. Poor metabolic control was found in $85 \%(51 / 60)$ of all participants; controlled blood pressure in $71.2 \%(42 / 60)$, controlled LDL-C levels in $10 \%(6 / 60)$, and controlled $\mathrm{HbA} 1 \mathrm{c}$ values in $48.3 \%$ (29/60) participants, respectively. Chronic complications such as retinopathy were found in $3.33 \%$ (2/60) participants, neuropathy in 10\% (6/60), nephropathy in 1.7\% (1/60), high blood pressure in 30\% (18/60) and cerebrovascular disease in $5 \%(3 / 60)$ of all participants. Conclusion: There is a high prevalence of poor metabolic control in patients with type 2 diabetes, despite being taken care of in a private clinic. It is necessary to take actions in order to improve the metabolic control in patients with type 2 diabetes attending the private health care sector.
\end{abstract}

Keywords: Diabetes mellitus, type 2; Health facilities, proprietary; Metabolism (source: MeSH NLM).

\section{INTRODUCCIÓN}

La diabetes mellitus tipo 2 (DM2), representa un grave problema de salud pública por la magnitud de costos y complicaciones que genera $^{[1,2]}$. Se ha calculado, que la DM2 produjo 1,5 millones de muertes en el año 2012, ocurriendo el $80 \%$ de estas muertes en países de bajos y medianos ingresos, donde se estima el mayor aumento en el número de casos para el año $2030^{[3,4]}$. En Latinoamérica y el Caribe la prevalencia global de DM2 es del $6,1 \%$ de acuerdo al reporte del $2014^{[5]}$. En algunas regiones de Perú la prevalencia de esta enfermedad es similar ${ }^{[6,7]}$.

Es conocido que el control de personas con DM2 basado únicamente en el valor de la glicemia no es suficiente para reducir la morbilidad y mortalidad provocada por la enfermedad, incluso pueden presentarse resultados adversos como hipoglicemia y mayor mortalidad ${ }^{[8,9]}$, a diferencia de un control integral (glicemia, lípidos y presión arterial) de la enfermedad, que sí ha demostrado reducción de eventos cardiovasculares y microvasculares, así como, un menor riesgo de muerte por causas o eventos cardiovasculares ${ }^{[10,11]}$. La American Diabetes Association (ADA) recomienda cumplir y usar como calidad de atención en diabetes, al control exitoso de los valores de hemoglobina glicosilada (HbA1c), presión arterial y la lipoproteína de baja densidad (LDL), como estrategia eficiente para lograr la disminución del riesgo del desarrollo de complicaciones crónicas ${ }^{[12]}$.

Asimismo, es importante señalar que existen otros factores que influyen en el control metabólico y calidad de vida de personas con DM2, los cuales son la adherencia al tratamiento ${ }^{[13]}$, actividad física ${ }^{[14]}$, autoeficacia y depresión, pero usualmente los médicos se centran en el manejo y control exhaustivo de la glucosa y no toman en cuenta los aspectos psicosociales de la enfermedad que tienen gran impacto en la situación de salud de la persona con DM2 y en la relación carga/capacidad, de gran importancia en la evaluación de la adherencia al tratamiento en enfermedades crónicas ${ }^{[15]}$. Por ello, es importante evaluar éstos factores y su asociación con el control de los parámetros laboratoriales en la población peruana.

Por otro lado, los sistemas de salud de Perú tanto públicos como privados no tienen un sistema de vigilancia y monitoreo de seguimiento obligatorio para personas con diabetes mellitus; $y$ aunque desde el año 2010 se encuentra disponible un Sistema de Vigilancia en Diabetes Mellitus en Perú, este solo funciona en algunos hospitales públicos, por lo que se desconoce esta información de los establecimientos de salud privados ${ }^{[16]}$, impidiendo el adecuado análisis de la situación de las personas con DM2 en este ámbito de atención.

La mayoría de estudios publicados en diabetes y control de la enfermedad han sido en hospitales públicos o con énfasis en la evaluación de la glicemia. En ese sentido, el objetivo de nuestro estudio fue determinar la calidad del control metabólico (HbA1c, presión arterial y LDL) y conocer la frecuencia de otros factores psicosociales como adherencia al tratamiento, depresión, calidad de vida, entre otros, en personas con DM2 en la consulta ambulatoria de una clínica privada, para tener una mirada del problema en el contexto de este segmento de atención y generar evidencia de posibles necesidades no cubiertas, que pueda servir para ajustar sus actuales parámetros de calidad de atención de la DM2.

\section{MATERIALES Y MÉTODOS}

\section{Diseño del estudio y participantes}

Se realizó un estudio de corte trasversal, en el cual se evaluó a personas con diagnóstico de DM2 con o sin seguro privado que acudieron al consultorio externo de endocrinología de la Clínica Médica Cayetano Heredia en Lima, Perú entre julio y diciembre del 2013. Ésta clínica se encuentra ubicada en el distrito de San Martín de Porres, situado al noroeste del centro de Lima, según la Superintendencia Nacional de Salud (SUSALUD), 
Tabla 1. Criterios para control glicémico, perfil lipídico y presión arterial según la American Diabetes Association.

\begin{tabular}{lc}
\multicolumn{1}{c}{ Criterio } & Valor \\
\hline $\begin{array}{l}\text { Para control glicémico } \\
\text { Hemoglobina glicosilada } \\
\text { Controlada } \\
\text { Glucosa basal controlada }\end{array}$ & $\leq 7,0 \%$ \\
Para perfil lipídico & Entre 70 y $130 \mathrm{mg} / \mathrm{dl}$ \\
Colesterol LDL controlado & $<100 \mathrm{mg} / \mathrm{dl}$ \\
Triglicéridos controlados & $<150 \mathrm{mg} / \mathrm{dl}$ \\
Colesterol HDL controlados & \\
Varón & $>40 \mathrm{mg} / \mathrm{dl}$ \\
Mujer & $>50 \mathrm{mg} / \mathrm{dl}$ \\
Para presión arterial & $<130 \mathrm{mmHg}$ \\
Presión arterial sistólica & $<80 \mathrm{mmHg}$ \\
Presión arterial diastólica & \\
\hline
\end{tabular}

está categorizado con nivel de complejidad II-1 ${ }^{[17,18]}$. Según el Instituto Nacional de Estadística e Informática (INEI) el distrito cuenta con una población de más de 673149 habitantes, el nivel socioeconómico de su población es entre medio y bajo.

Los criterios de inclusión fueron: tener diagnóstico de DM2 con un tiempo de enfermedad mayor de 12 meses, edad de 18 años o mayor, haber asistido a consulta al menos una vez en el último año, que puedan leer y entender el idioma español o no. Fueron excluidos del estudio las personas diagnosticados de diabetes secundaria según criterios de la ADA ${ }^{[19]}$, personas que padezcan alguna enfermedad crónica no metabólica (lupus eritematoso sistémico, artritis reumatoide, EPOC, entre otras), personas hospitalizadas en los últimos 6 meses o personas que hayan sufrido complicaciones mayores de la DM2 (cetoacidosis diabética, estado hiperosmolar, enfermedad cerebrovascular, infarto de miocardio y/o pie diabético amputado) en el último año, o que no brindaron su consentimiento de participación en el estudio.

\section{Procedimientos}

Luego de la firma del consentimiento informado, se procedió a completar el cuestionario basal con datos sociodemográficos y clínicos mediante una entrevista cara a cara con el participante, así como la "Escala de Adherencia al Tratamiento en Diabetes Mellitus 2, versión III" (EATDM-IIIC), el “Cuestionario Internacional de Actividad Física" (IPAQ), El "Instrumento de Evaluación de Calidad de Vida en el Paciente Diabético" (Diabetes-39), el "Cuestionario de Autoeficacia de Schwarzer y Baessler", y la "Escala de Depresión del centro de estudios epidemiológicos" (CES-D 20). Además, se realizó la toma de presión arterial, peso, talla y circunferencia abdominal.

Se obtuvo una muestra de sangre al terminar la entrevista, realizada por un personal capacitado y cumpliendo las normas de bioseguridad. Los participantes debieron cumplir un mínimo de ocho horas de ayuno para que se considere como válidas las tomas de muestra, en caso el participante no estaba en ayunas se le citó nuevamente dentro de la semana posterior a la fecha de reclutamiento.

\section{Evaluación y medidas de resultados}

Se tomaron en cuenta los criterios descritos en la Tabla 1 para el control glicémico, perfil lipídico y presión arterial ${ }^{[19]}$.

Se calificó como control metabólico adecuado a aquellos participantes que cumplieron con los criterios de hemoglobina glicosilada, presión arterial y LDL controlados. Si no cumplían los criterios se consideraba que el participante tenía pobre control metabólico. Se consideraron factores potenciales relacionados al mal control metabólico como tiempo de enfermedad ( $\geq 10$ años y $<10$ años de enfermedad), índice de masa corporal clasificado como IMC; normal $(18,5-<25 \mathrm{~kg} /$ $\left.\mathrm{m}^{2}\right)$, sobrepeso $\left(\geq 25-<30 \mathrm{~kg} / \mathrm{m}^{2}\right)$, obesidad tipo $1(\geq 30-<35$ $\left.\mathrm{kg} / \mathrm{m}^{2}\right)$ y obesidad tipo $2\left(\geq 35 \mathrm{~kg} / \mathrm{m}^{2}\right)$ y circunferencia abdominal ( $\leq 88$ para mujeres $\mathrm{y} \leq 102$ para varones como valores normales) ${ }^{[19]}$. Se identificó además la presencia de las complicaciones más comunes de la diabetes, por autorreporte, considerando complicaciones microvasculares (nefropatía, neuropatía, y retinopatía) y complicaciones macrovasculares (accidente cerebrovascular, enfermedad vascular periférica e infarto de miocardio agudo). Se tomó en cuenta también el tratamiento recibido, hipoglicemiantes orales, insulina, e insulina más hipoglicemiantes orales. Adicionalmente se midió la adherencia al tratamiento utilizando la escala EATDM-IIIC. La escala tiene un máximo resultado posible de $100 \%$, considerándose como buena adherencia valores mayores al $66 \%{ }^{[20]}$.

Para medir actividad física se utilizó el protocolo International Physical Activity Questionnaire (IPAQ). Se consideró al participante como 1) activo, si realizó actividad de vigorosa intensidad al menos 3 días con una acumulación mínima de 1500 MET (múltiplos de la tasa metabólica en reposo) min/semana, 7 o más días de caminata, actividad moderada, 0 actividades de intensidad vigorosa, acumulando como mínimo 3000 MET min/ semana. 2) Mínimamente activo: si tiene 3 o más días de actividad vigorosa o al menos 20 minutos de actividad por día, 5 o más días de actividad de moderada intensidad y/o caminata de al menos 30 minutos por día, 5 o más días de cualquier combinación de caminata o actividad vigorosa que alcancen al menos 600 MET $\mathrm{min} /$ semana. 3) Inactivo: si no existe actividad reportada, o si es reportada alguna actividad física no suficiente para corresponder a las categorías 1 o $2^{[21]}$

Se utilizó el instrumento Diabetes-39, que evalúa la calidad de vida en personas con diabetes mellitus en 39 ítems divididos en 5 secciones (Energía-Movilidad, Control de la Diabetes, Ansiedad-Preocupación, Carga Social y Funcionamiento Sexual). Además, contiene dos ítems finales que califican la percepción del participante acerca de su calidad de vida de manera global y la severidad de la diabetes, los cuáles no serán considerados en este estudio. Las puntuaciones parciales de cada sección y la 
Tabla 2. Características clínicas $(n=60)$.

\begin{tabular}{|c|c|}
\hline Característica & $n(\%)$ \\
\hline \multicolumn{2}{|l|}{ Índice de masa corporal } \\
\hline Normal & $23(38,3)$ \\
\hline Sobrepeso & $21(35,0)$ \\
\hline Obesidad tipo I & $13(21,7)$ \\
\hline Obesidad tipo II & $3(5,0)$ \\
\hline \multicolumn{2}{|l|}{ Tiempo de diagnóstico } \\
\hline Más de 10 años & $18(30,0)$ \\
\hline Menos de 10 años & $42(70,0)$ \\
\hline \multicolumn{2}{|c|}{ Tratamiento para diabetes mellitus tipo 2} \\
\hline Hipoglicemiantes orales & $46(76,6)$ \\
\hline Insulina & $1(1,7)$ \\
\hline Hipoglicemiantes + insulina & $12(20,0)$ \\
\hline Sin medicación & $1(1,7)$ \\
\hline \multicolumn{2}{|l|}{ Retinopatía } \\
\hline No & $58(96,7)$ \\
\hline $\mathrm{Si}$ & $2(3,3)$ \\
\hline \multicolumn{2}{|l|}{ Neuropatía Diabética } \\
\hline No & $54(90,0)$ \\
\hline $\mathrm{Si}$ & $6(10,0)$ \\
\hline \multicolumn{2}{|l|}{ Nefropatía Diabética } \\
\hline No & $59(98,3)$ \\
\hline $\mathrm{Si}$ & $1(1,7)$ \\
\hline \multicolumn{2}{|l|}{ Accidente cerebrovascular } \\
\hline No & $57(95,0)$ \\
\hline $\mathrm{Si}$ & $3(5,0)$ \\
\hline \multicolumn{2}{|l|}{ Enfermedad vascular periférica } \\
\hline No & $59(98,3)$ \\
\hline $\mathrm{Si}$ & $1(1,7)$ \\
\hline \multicolumn{2}{|l|}{ Infarto de miocardio } \\
\hline No & $58(96,7)$ \\
\hline $\mathrm{Si}$ & $2(3,3)$ \\
\hline \multicolumn{2}{|l|}{ Hipertensión Arterial } \\
\hline No & $42(70,0)$ \\
\hline $\mathrm{Si}$ & $18(30,0)$ \\
\hline
\end{tabular}

*IMC: Índice de masa corporal

puntuación total de calidad de vida se transforman en una escala del 0 al 100 donde 0 es para nada afectado y 100 muy afectado. Los resultados no presentan un punto de corte definido, siendo utilizados en términos de comparación con otras muestras ${ }^{[22]}$.

El cuestionario de autoeficacia de Schwarzery Baessler consta de 10 ítems con cuatro opciones de respuesta: "incorrecto", "apenas cierto", "más bien cierto" y "cierto". Se asigna un valor de 1 al 4 a cada uno de los ítems. A mayor puntaje, mayor autoeficacia percibida. No existiendo un punto de corte para definir si una persona se considera autoeficaz o no; se compara medias de autoeficacia entre grupos para definirlo ${ }^{[23]}$.

Para evaluar depresión se usó el CES-D20, las respuestas están basadas en la frecuencia de ocurrencia de sintomatología depresiva durante la semana pasada a la entrevista. Ésta usa una escala ordinal de 4 puntos: raramente o nunca (menos de 1 día), algunas o pocas veces (de 1 a 2 días), ocasionalmente o por un moderado periodo de tiempo (de 3 a 4 días), la mayor parte o todo el tiempo (de 5 a 7 días). Un puntaje máximo de 16 es indicativo de sintomatología depresiva leve o significativa, puntajes mayores a 16 indican sintomatología depresiva severa ${ }^{[24]}$.

\section{Procedimientos de laboratorio}

Las muestras de sangre fueron procesadas para la obtención de HbA1c, glucosa capilar, colesterol, triglicéridos, LDL y lipoproteínas de alta densidad (HDL).

\section{Métodos estadísticos}

El tamaño de muestra calculado fue de 60 participantes, Para la determinación del tamaño de la muestra se asumió una prevalencia del control metabólico adecuado de $15 \%{ }^{[25]}$, con un nivel de confianza $95 \%$ y una precisión del $9 \%$. Los datos obtenidos fueron ingresados a una hoja de cálculo de Microsoft Excel 2013 y luego transferidos al programa Stata 12.0 (StataCorp, College Station, TX, USA) donde fueron analizados. Se calcularon medidas de tendencia central y dispersión, como media y desviación estándar (DE) para variables cuantitativas, y porcentajes e intervalos de confianza al 95\% (IC95\%) para variables categóricas. Para la evaluación de factores asociados a control metabólico se usó test t-Student o Chi cuadrado de Pearson. Para comparar medias de variables paramétricas se utilizó el test t-Student. El test de Chi cuadrado de Pearson se usó para comparar variables categóricas y la prueba exacta de Fisher en caso la frecuencia esperada era menor a cinco. En todos los casos se consideró que las diferencias eran significativas si el valor de $p \leq 0,05$.

\section{Declaraciones éticas}

El comité Institucional de ética del Hospital Cayetano Heredia aprobó el protocolo del estudio y se contó además con la aprobación de la Dirección de la Clínica Médica Cayetano Heredia donde se realizó la recolección de datos. Además, se obtuvo consentimientos informados por escrito de todos los participantes.

\section{RESULTADOS}

\section{Características demográficas}

El tamaño de la muestra fue de 60 participantes. No hubo abandonos. El 53,3\% (32/60) fue de sexo femenino y la media de edad fue $63,1 \pm 13,2$ años. De los participantes, $66,6 \%$ (40/60) tenían pareja. 
Tabla 3. Resultados de laboratorio

\begin{tabular}{lc}
\multicolumn{1}{c}{ Característica } & $\begin{array}{c}\text { Media } \pm \text { desviación } \\
\text { estándar }\end{array}$ \\
\hline Glucosa basal (mg/dL) & $141,3 \pm 52,3$ \\
Hemoglobina glicosilada (\%A1C) & $7,7 \pm 2,0$ \\
Colesterol total (mg/dL) & $191,1 \pm 36,8$ \\
Colesterol LDL* (mg/dL) & $109,7 \pm 26,7$ \\
Colesterol HDL+ (mg/dL) & $47,9 \pm 11,4$ \\
Triglicéridos (mg/dL) & $168,0 \pm 82,4$ \\
\hline
\end{tabular}

*LDL: Low-density lipoprotein.

tHDL: High-density lipoprotein.

\section{Características clínicas}

El tiempo medio de diagnóstico de DM2 fue $8,1 \pm 6,2$ años, teniendo el $30 \%(18 / 60)$ de participantes más de 10 años de diagnóstico (ver Tabla 2).

En cuanto al tratamiento, $1,7 \%(1 / 60)$ de los participantes recibían solo tratamiento con insulina, 76,6\% (46/60) recibían solo hipoglicemiantes orales, $20 \%$ (12/60) recibía terapia combinada de insulina más hipoglicemiantes orales y $1,6 \%$ (1/60) no recibía ningún tipo de medicación (ver Tabla 2).

Respecto a las complicaciones $18,3 \%$ (11/60) de los participantes manifestaron al menos una complicación de la diabetes, se autorreportó retinopatía en el 3,3\% (2/60), neuropatía en el $10 \%(6 / 60)$, nefropatía en el $1,7 \%(1 / 60)$ y $5 \%(3 / 60)$ reportaron antecedente de accidente cerebrovascular. Sólo el 1,7\% $(1 / 60)$ reportó enfermedad vascular periférica y 3,3\% (2/60) infarto de miocardio. Por otro lado, hipertensión arterial fue autorreportado por el $30 \%$ (18/60) de los participantes (ver Tabla 2).

Además, se encontró que el 21\% (35/60) participantes de la muestra tuvieron sobrepeso, $21,7 \%$ (13/60) obesidad tipo 1, el $5 \%$ (3/60) índice de obesidad tipo 2 y solo $38,3 \%(23 / 60)$ de los participantes se encontraban dentro del rango normal (ver Tabla 2). El $53,3 \%$ del total de participantes se encontraba dentro de los valores normales de circunferencia abdominal.

\section{Resultados clínicos y control metabólico}

Según los objetivos terapéuticos propuestos por la ADA, 48,3\% (29/60) de los participantes tenían valores de HbA1C controlada y $53,3 \%(32 / 60)$ tenían glicemia basal controlada. Se encontró colesterol LDL y triglicéridos controlados en $33,3 \%$ (20/60) y $50 \%$ $(30 / 60)$ de los participantes respectivamente. Solo $10 \%(6 / 60)$ tuvieron un perfil lipídico adecuado según recomendaciones de la ADA (ver Tabla 3).

En cuanto al control metabólico solo el 15\% (9/60) de participantes cumplieron los criterios de la ADA de un buen control (ver Tabla 4).

\section{Factores asociados al control metabólico}

Según la escala de adherencia EATDM-IIIC solo el 18,3\% (11/60) de los participantes tenían una buena adherencia al tratamiento, según los resultados del cuestionario de actividad física IPAQ el $53,3 \%(32 / 60)$ de los participantes se encontraban físicamente activos; mientras que $33,3 \%(20 / 60)$ y $13,3 \%(8 / 60)$ estaban mínimamente activos e inactivos respectivamente.

La mediana y rango intercuartílico (RIC) del cuestionario de calidad de vida (Diabetes-39) fue de 33,9 (26,1-45,9), mientras que la media y desviación estándar de resultados del cuestionario de autoeficacia (Cuestionario de autoeficacia de Schwarzer y

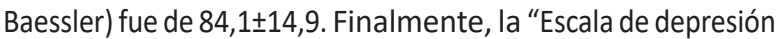
CES-D20 indicó que el 30\% (18/60) de los participantes del estudio tenían sintomatología depresiva. No se encontró asociación de ninguno de los resultados anteriores con un adecuado control glicémico (ver Tabla 5).

\section{DISCUSIÓN}

Nuestros hallazgos sugieren que las personas con DM2 atendidas en esta clínica privada tienen una elevada frecuencia de control metabólico inadecuado, estando el $85 \%$ mal controlados de acuerdo con los criterios de la ADA. Este hallazgo es similar al de otras poblaciones estudiadas, en la India un estudio realizado en población reportó un $86,4 \%$ de control metabólico inadecuado en sus participantes ${ }^{[25]}$; asimismo, un estudio realizado en un centro de salud público en España, encontró que de su muestra sólo el 9,4\% (IC 95\%: 5-15,5\%) de varones y el 5\% (IC 95\%: 2,2$9,6 \%)$ de mujeres cumplía con los controles de glucemia basal, hemoglobina glicosilada, presión arterial sistólica y diastólica, colesterol LDL y tabaquismo ${ }^{[26]}$. Por otro lado, un estudio en un establecimiento público de Perú, halló un 92,5\% de control metabólico inadecuado ${ }^{[27]}$.

Los resultados mostrados, provenientes de estudios realizados en países de altos, medianos y bajos ingresos, indicarían que el mal control metabólico es un problema global. Un estudio descriptivo retrospectivo en España desarrollado en personas con DM2 de una clínica privada, seguidos por un año en consulta externa de medicina interna, muestra en sus resultados un logro de control metabólico integral: presión arterial sistólica $<130$, presión arterial diastólica $<80$, colesterol LDL $<99$ y HbA1c $<7 \%$, en el $26,2 \%$ de los participantes, lográndose los objetivos en un rango superior a lo reportado en otros estudios (13,6\%, 9,4\%, $5 \%$ y $7,5 \%)^{[28]}$. Lo que demuestra, que interviniendo y haciendo seguimiento de estos parámetros, se puede lograr mejoras en ellos.

En cuanto a los factores relacionados al buen o mal control metabólico, nuestro estudio arrojó una media global de la escala EATDM-III de 54,6\% similar a la media de adherencia de 60,3\% de un estudio realizado en Costa Rica ${ }^{[29]}$. Existen escalas dirigidas a conocer solo si el participante consume o no los fármacos ${ }^{[30]}$, la 
ventaja de la EATDM-III se encuentra en la evaluación de otros ítems ligados estrechamente a la adherencia al tratamiento, como: apoyo familiar, organización y apoyo comunal, ejercicio físico, control médico, higiene y autocuidado, la dieta y la valoración de la actividad física. La adherencia al tratamiento representa un desafío para el control metabólico adecuado de la persona con DM2, pues resulta difícil su cumplimiento, debiendo tomarse en cuenta como parte del control integral.

Por otro lado, los resultados del IPAQ indicaron que el 53,3\% de los participantes en el estudio se encontraban físicamente activos, mientras que el $33,3 \%$ y $13,3 \%$ estaban mínimamente activos e inactivos respectivamente, nuestros resultados difieren de un estudio en Perú en el cuál $12 \%$ de los participantes de la muestra estaban físicamente activos, $68 \%$ mínimamente activos y el $20 \%$ inactivos ${ }^{[31]}$. Estas diferencias se pueden deber a la variedad del contexto de atención, ya que el segundo estudio se realizó en un hospital público; en nuestro país el acceso a hospitales públicos o privados muchas veces es indicador de disparidades económicas, laborales e incluso de hábitos saludables. En Colombia, Figueroa reportó, de una muestra de 111 personas con prediabetes, que el $53,2 \%$ de los participantes eran físicamente activos, mientras que $46,8 \%$ fueron mínimamente activos e inactivos ${ }^{[32]}$, asemejándose a nuestros resultados, posiblemente debido a que el $55,9 \%$ de los participantes de ese estudio eran de estrato socioeconómico medio, medio alto y alto, además la media de edad fue de 51 años, incrementando la posibilidad de actividad en los individuos de esta muestra ${ }^{[33]}$. Es importante incorporar el seguimiento de la actividad física de la persona con DM2, antes y durante su tratamiento, pues se ha demostrado su efecto positivo sobre los parámetros de control metabólico, convirtiéndola en parte fundamental del tratamiento de la DM2 ${ }^{[34]}$.

La mediana del cuestionario Diabetes-39 fue de 33,9 (RIC: $26,1-45,9)$, contrastando con un estudio de Brasil donde la mediana de calificación total para la muestra fue de $43,5^{[35]}$. La calidad de vida resultó mejor en nuestra muestra, posiblemente debido al porcentaje de personas con un tiempo de enfermedad mayor a 10 años, el cual era de $30 \%$ para nuestro estudio y de $46,6 \%$ para el estudio de Brasil. Por otro lado, hay evidencia que sugiere que una terapia multifactorial (hemoglobina glicosilada, presión arterial, colesterol LDL y triglicéridos) en las personas con DM2, aparte de mejorar el control de estos parámetros puede aumentar su calidad de vida, lo que refuerza la idea de un enfoque más amplio de control metabólico ${ }^{[36]}$.

El cuestionario de autoeficacia de Schwarzer y Baessler, dio una media de resultados de $84 \pm 15$, estudios en la población general en Latinoamérica muestran resultados similares; un estudio aplicando el mismo cuestionario realizado en población general de Chile en individuos entre 15 y 65 años obtuvo una media de $85,5 \pm 4,84{ }^{[37]}$. En nuestro estudio, el nivel de autoeficacia reportado por los participantes llama la atención por ser muy alto, casi igualando al nivel de autoeficacia de la población general en Chile; creemos que la explicación está en el alto grado de independencia que llegan a adoptar algunos pacientes en
Tabla 4. Control metabólico en pacientes con diabetes mellitus tipo 2 de una clínica privada $\mathrm{N}=60$.

\begin{tabular}{lc}
\multicolumn{1}{c}{ Variable } & $\mathbf{n}(\%)$ \\
\hline $\begin{array}{l}\text { Hemoglobina glicosilada } \\
\text { Controlada }\end{array}$ & $29(48,3)$ \\
No controlada & $31(51,7)$ \\
Colesterol LDL* & \\
Controlado & $20(33,3)$ \\
No controlado & $40(66,7)$ \\
Presión arterial & \\
Controlada & $42(71,2)$ \\
No controlada & $17(28,8)$ \\
Control Metabólico & \\
Controlado & $9(15,0)$ \\
No controlado & $51(85,0)$ \\
\hline
\end{tabular}

*LDL: Low-density lipoprotein

nuestro país, pues la situación económica, obliga a los familiares y allegados de estos pacientes a trabajar durante casi todo el día.

El 30\% de la muestra de nuestro estudio padecía de síntomas depresivos, un estudio en China en personas con DM2 muestra resultados consistentes con los nuestros identificando con depresión a $31 \%$ de los participantes ${ }^{[38]}$. Otra investigación realizada en jóvenes con diabetes empleando el CES-D encontró relación entre el mal control glicémico y la presencia de síntomas depresivos moderados a severos, reportando además que un $22,6 \%$ de los participantes de ese estudio tenían síntomas depresivos ${ }^{[39]}$.

Como limitaciones del estudio debemos señalar que el pequeño tamaño de muestra no permite hacer comparaciones con otras realidades, además no son extrapolables los resultados a toda la población de pacientes del sector privado con DM2, asimismo no nos permite establecer asociaciones entre las variables psicosociales contempladas y los parámetros de control metabólico. Algunas de las escalas utilizadas en nuestro estudio no tienen puntos de corte ("Diabetes-39", y "Cuestionario de Autoeficacia de Schwarzer y Baessler"), siendo sólo útiles para comparar resultados con otros estudios, pues no indican la presencia o no de adecuados o inadecuadas medidas de estos parámetros en la muestra. Sin embargo, se lograron reportar resultados que reflejan un acercamiento al estado del control metabólico en personas con DM2 en las clínicas privadas; además, la recolección de datos de complicaciones a través del autorreporte puede estar subestimando la prevalencia de esos resultados y validez del estudio obtención de datos de complicaciones de la diabetes a través del autorreporte de los pacientes puede estar subestimando la prevalencia de esos resultados y validez del estudio. La fortaleza de nuestro estudio, radica en ser de los pocos estudios realizados en el sector privado, ámbito de atención usado por un $2 \%$ de usuarios de sistemas de salud en nuestro país ${ }^{[40]}$, que pese a reportar mejores resultados 
Tabla 5. Factores asociados al control metabólico.

\begin{tabular}{lccc}
\multicolumn{1}{c}{ Variable } & $\begin{array}{c}\text { Hemoglobina glicosilada } \\
\text { controlada } \\
\mathbf{N}=\mathbf{2 9}\end{array}$ & $\begin{array}{c}\text { Hemoglobina glicosilada no } \\
\text { controlada } \\
\mathbf{N = 3 1}\end{array}$ & Valor $\mathbf{p}$ \\
\hline $\begin{array}{l}\text { IPAQ, } \mathrm{n}(\%) \\
\text { Inactivo }\end{array}$ & $6(75,0 \%)$ & $2(25,0 \%)$ & $0,24 *$ \\
Mínimamente activo & $8(40,0 \%)$ & $12(60,0 \%)$ & $17(53,1 \%)$ \\
Activo & $15(46,9 \%)$ & & $0,69 \dagger$ \\
Depresión, n (\%) & $8(44,4 \%)$ & $10(55,6 \%)$ & $21(50,0 \%)$ \\
Si & $21(50,0 \%)$ & $53,0(10,0)$ & $0,27 \ddagger$ \\
No & $56,0(9,0)$ & $40,0(20,0)$ & $0,60 \ddagger$ \\
Adherencia, media (DE) & $33,0(15,0)$ & $82,0(17,0)$ & $0,13 \ddagger$ \\
Calidad de vida, media (DE) & $86,0(13,0)$ & & \\
Autoeficacia, media (DE) & & \\
\hline
\end{tabular}

IPAQ: “Cuestionario Internacional de Actividad Física” (International Physical Activity Questionnaire)

DE: Desviación estándar

* Prueba exacta de Fisher, † Prueba de Chi cuadrado, $¥$ Test de $t$ de student

que en el caso del sector público en cuanto al control metabólico, este continúa siendo elevado.

En conclusión, no se estaría realizando un control metabólico adecuado en la población con DM2 de esta clínica privada, por lo cual es esperable la aparición de complicaciones crónicas de esta enfermedad traducidas en gran carga económica a nivel familiar y del sistema de salud. Los resultados obtenidos brindan un alcance del estado actual de control metabólico en una clínica privada de Lima, cuyo valor reportado es preocupante, en ese sentido, es necesario hacer un monitoreo y seguimiento en instituciones públicas y privadas para promover la adopción de una evaluación integral de la persona con DM2, según las recomendaciones brindadas por la ADA y adherirse a estas recomendaciones a la hora de evaluar la calidad de atención en diabetes, lo que debería hacerse de manera institucionalizada. Asimismo, a crear conciencia de que el control metabólico adecuado no sólo responde a una normalización de los parámetros laboratoriales, sino que se ve regulado además por múltiples factores psicosociales.

\section{Fuente de financiamiento: autofinanciado.}

Conflictos de interés: Los autores declara no tener conflictos de interés.

\section{REFERENCIAS BIBLIOGRÁFICAS}

1. Arredondo A. Type 2 diabetes and health care costs in Latin America: exploring the need for greater preventive medicine. BMC Medicine. 2014;12(1):136

2. Seuring T, Archangelidi O, Suhrcke M. The economic costs of type 2 diabetes: a global systematic review. Pharmacoeconomics. 2015;33(8):811-31.
3. Whiting DR, Guariguata L, Weil C, Shaw J. IDF diabetes atlas: global estimates of the prevalence of diabetes for 2011 and 2030. Diabetes Res Clin Pract. 2011;94(3):311-21.

4. Mathers CD, Loncar D. Projections of global mortality and burden of disease from 2002 to 2030. PLoS medicine. 2006;3(11):e442.

5. Aschner P, Aguilar-Salinas C, Aguirre L, Franco L, Gagliardino JJ, de Lapertosa SG, et al. Diabetes in South and Central America: an update. Diabetes research and clinical practice. 2014;103(2):238-43.

6. Medina-Lezama J, Zea-Diaz H, Morey-Vargas OL, Bolanos-Salazar JF, Munoz-Atahualpa E, Postigo-MacDowall M, et al. Prevalence of the metabolic syndrome in Peruvian Andean hispanics: the prevencion study. Diabetes Res Clin Pract. 2007;78(2):270-81.

7. Miranda JJ, Gilman RH, Smeeth L. Differences in cardiovascular risk factors in rural, urban and rural-to-urban migrants in Peru. Heart. 2011;97(10):787-96.

8. Gerstein HC, Miller ME, Byington RP, Goff DC, Jr., Bigger JT, Buse $\mathrm{JB}$, et al. Effects of intensive glucose lowering in type 2 diabetes. N Engl J Med. 2008;358(24):2545-59.

9. The ACCORD Study Group. Long-term effects of intensive glucose lowering on cardiovascular outcomes. N Engl J Med. 2011;364(9):818-28.

10. Gaede P, Lund-Andersen $\mathrm{H}$, Parving HH, Pedersen O. Effect of a multifactorial intervention on mortality in type 2 diabetes. $\mathrm{N} \mathrm{Engl}$ J Med. 2008;358(6):580-91.

11. Gaede P, Vedel P, Larsen N, Jensen GV, Parving HH, Pedersen $O$. Multifactorial intervention and cardiovascular disease in patients with type 2 diabetes. N Engl J Med. 2003;348(5):383-93.

12. American Diabetes Association. Standards of medical care for patients with diabetes mellitus. Diabetes Care. 2002;25(1):16.

13. García-Pérez LE, Álvarez M, Dilla T, Gil-Guillén V, Orozco-Beltrán D. Adherence to therapies in patients with type 2 diabetes. Diabetes Ther. 2013;4(2):175-94.

14. Gong $\mathrm{QH}$, Kang JF, Ying YY, Li H, Zhang XH, Wu YH, et al. Lifestyle interventions for adults with impaired glucose tolerance: a systematic review and meta-analysis of the effects on glycemic control. Intern Med. 2015;54(3):303-10.

15. Bodde AE, Shippee ND, May CR, Mair FS, Erwin PJ, Murad MH, et al. Examining health promotion interventions for patients with chronic conditions using a novel patient-centered complexity model: protocol for a systematic review and meta-analysis. Syst Rev. 2013;2:29. 
16. Revilla L. Situación de la vigilancia de diabetes en el Perú, al I semestre de 2013. Bol epidemiol. 2013;22(39):4.

17. Superintendencia Nacional de Salud. Listado de establecimientos registrados en el Renipress [Internet]. Lima, Perú: SuSalud; 2015 [citado el 05 de julio de 2017]. Disponible en: http:// app20.susalud.gob.pe:8080/registro-renipress-webapp/ listadoEstablecimientosRegistrados.htm?action=mostrarBuscar\#noback-button

18. Ministerio de Salud. NTS N021-MINSA/dgsp-v.03. Categoría de establecimientos del sector salud [Internet]. Lima, Perú: MINSA; 2011 [citado el 05 de julio de 2017]. Disponible en: http://www.digemid.minsa.gob.pe/UpLoad/UpLoaded/PDF/ AtencionFarmaceutica/Categorizacion-UPSS_Farmacia.pdf

19. American Diabetes Standards of Medical Care in Diabetes-2015: Summary of Revisions. Diabetes Care. 2015; 38 (Supplement 1): S4.

20. Villalobos A, Brenes JC, Quirós D, León G. Características psicométricas de la Escala de Adherencia al Tratamiento de la Diabetes Mellitus Tipo II - Versión III (EATDM-IIIC) en una muestra de pacientes diabéticos de Costa Rica. Act Col de Psico. 2006;9:31-8.

21. Mantilla Toloza SC, Gómez-Conesa A. El Cuestionario Internacional de Actividad Física. Un instrumento adecuado en el seguimiento de la actividad física poblacional. Rev Ibero de Fis y Kine. 2007;10(01):48-52.

22. López JM, Rodríguez R. Adaptación y validación del instrumento de calidad de vida Diabetes 39 en pacientes mexicanos con diabetes mellitus tipo 2. Salud Publica Mex. 2006;48(3):200-8.

23. Cid HP, Orellana A, Barriga O. Validación de la escala de autoeficacia general en Chile. Rev Med Chil. 2010;138:551-7.

24. Center for Substance Abuse Treatment. Managing depressive symptoms in substance abuse clients during early recovery [Internet]. Rockville (MD): Substance Abuse and Mental Health Services Administration (US); 2008 [citado el 05 de julio de 2017]. Disponible en: https://www.ncbi.nlm.nih.gov/books/NBK64057/

25. Raman R, Gupta A, Kulothungan V, Sharma T. Prevalence and risk factors of diabetic retinopathy in subjects with suboptimal glycemic, blood pressure and lipid control. Sankara Nethralaya Diabetic Retinopathy Epidemiology and Molecular Genetic Study (SN-DREAMS, Report 33). Curr Eye Res. 2012;37(6):513-23.

26. Domínguez Sánchez-Migallón P. Control Metabólico en Pacientes Diabéticos Tipo 2: grado de Control y nivel de Conocimientos (Estudio AZUER). Rev Clín de Med de Fam. 2011;4:32-41.

27. Huayanay-Espinoza IE, Guerra-Castanon F, Lazo-Porras M, Castaneda-Guarderas A, Thomas NJ, Garcia-Guarniz AL, et al. Metabolic control in patients with type 2 diabetes mellitus in a public hospital in Peru: a cross-sectional study in a low-middle income country. PeerJ. 2016;4:e2577.

28. Sánchez M, Puerta S, Molero E, Ramos C, Buonaiuto V, Miralles F. Grado de control en pacientes con diabetes mellitus tipo 2 en consulta de Medicina Interna en un centro hospitalario privado. Rev Clínica Española. 2012;33.

29. Villalobos-Pérez A, Brenes-Sáenz J, Quirós-Morales D, LeónSanabria G. Características psicométricas de la escala de adherencia al tratamiento de la diabetes mellitus tipo ii-versión III (EATDM-IIIC) en una muestra de pacientes diabéticos de Costa Rica. Act Col de Psico. 2006;9:31-8.

30. Morisky DE, Ang A, Krousel-Wood M, Ward HJ. Predictive validity of a medication adherence measure in an outpatient setting. $J$ Clin Hypertens (Greenwich). 2008;10(5):348-54.

31. Manzaneda AJ, Lazo-Porras M, Málaga G. Actividad física en pacientes ambulatorios con diabetes mellitus 2 de un hospital nacional del Perú. Rev Peru Med Exp Salud Publica. 2015;32(2):311-5.

32. Figueroa FN, Morales J, Melgarejo A, Forero J, Gabriel M, León JA, et al. Characterization of patients with pre-diabetes in first-level health care service institutions Cali, Colombia. Colomb Med. 2011;42(1):98-106

33. Milanovic Z, Pantelic S, Trajkovic N, Sporis G, Kostic R, James N. Age-related decrease in physical activity and functional fitness among elderly men and women. Clin Interv Aging. 2013;8:549-56.

34. Colberg SR, Sigal RJ, Fernhall B, Regensteiner JG, Blissmer BJ, Rubin RR, et al. Exercise and type 2 diabetes: The American College of Sports Medicine and the American Diabetes Association: joint position statement. Diabetes Care. 2010;33(12):e147-67.

35. Zulian LR, Santos MAd, Veras VS, Rodrigues FFL, Arrelias CCA, Zanetti ML. Qualidade de vida de pacientes com diabetes utilizando o instrumento Diabetes 39 (D-39). Rev Gaucha Enferm. 2013;34(3):138-46

36. Menard J, Payette $H$, Baillargeon JP, Maheux P, Lepage S, Tessier $D$, et al. Efficacy of intensive multitherapy for patients with type 2 diabetes mellitus: a randomized controlled trial. CMAJ. 2005;173(12):1457-66.

37. Cid. H. Patricia, Y. Alda Orellana, O. Barriga. Validación de la escala de autoeficacia general en Chile. Rev Med Chil. 2010;138(5):5517

38. Zhang $\mathrm{Y}$, Ting RZW, Lam MHB, Lam SP, Yeung RO, Nan H, et al. Measuring depression with CES-D in Chinese patients with type 2 diabetes: the validity and its comparison to PHQ-9. BMC Psychiatry [Internet]. 2015;15 [citado el 05 de julio de 2017]. Disponible en: https://bmcpsychiatry.biomedcentral.com/ track/pdf/10.1186/s12888-015-0580-0?site=bmcpsychiatry. biomedcentral.com

39. Lawrence JM, Standiford DA, Loots B, Klingensmith GJ, Williams $D E$, Ruggiero $A$, et al. Prevalence and correlates of depressed mood among youth with diabetes: the search for diabetes in youth study. Pediatrics. 2006;117(4):1348-58.

40. Ministerio de Salud. Análisis de situación de salud del Perú. Lima, Perú: MINSA; 2013 